\title{
SKP2 Promotes Hepatocellular Carcinoma Progression Through Nuclear AMPK- SKP2-CARM1 Signaling Transcriptionally Regulating Nutrient-Deprived Autophagy Induction
}

\author{
Xiaoshuang Wei $e^{a, b} \quad$ Xu Li,b Wei Yan ${ }^{a, b} \quad$ Xinghua Zhang ${ }^{a, b} \quad$ Yu Sun ${ }^{a, b}$ \\ Feng Zhang ${ }^{a, b}$
}

aDepartment of Liver Surgery/Liver Transplantation Center, The First Affiliated Hospital of Nanjing

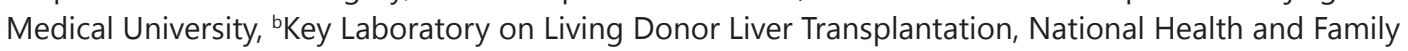
Planning Commission of the People's Republic of China, Nanjing, Jiangsu Province, P.R China

\section{Key Words}

SKP2 • CARM1 • Autophagy • AMPK • Hepatocellular Carcinoma

\begin{abstract}
Background/Aims: SKP2 overexpression has been associated with poor prognosis in numerous cancers. The mechanisms of autophagy in the tumor pathogenesis have been a research focus recently. How the SKP2 involved in autophagy expresses oncogenic characteristics, especially in HCC, are largely unclear. Methods: The expression of SKP2 was detected by qPCR, Western blot, Immunohistochemical (IHC) and Immunofluorescence (IF) techniques. SKP2 was knocked down or overexpressed by lentivirus transfection in HCC cells. Functional assays such as CCK8 assays, transwell migration and invasion assays, and colony formation assays were performed to determine the role of SKP2 in HCC. Furthermore, autophagy was induced by glucose deprivation in HCC cells followed by monitoring of the levels and distributions of SKP2, CARM1 and AMPK. Results: Our data showed that SKP2 levels were significantly increased in HCC cell lines and HCC tissues rather than corresponding normal liver tissues, and augmented SKP2 levels were statistically correlated with tumor grade, size and metastases. By up-regulation or down-regulation of SKP2 in HCC cells, we confirmed that SKP2 encourages proliferation, migration, invasion, and colony formation. We then found that SKP2 was inhibited, CARM1 increased and AMPK $\alpha 2$ became activated in the nucleus under glucose deprivation induced autophagy. Moreover, we discovered that SKP2 was repressing CARM1 in the nucleus under nutrient-sufficient conditions in HCC. Conclusions: We show that SKP2 promotes HCC progression and its nuclear functions of autophagy induction with CARM1 and AMPK, which may provide a potential target for HCC therapy.

Xiaoshuang Wei and Xu Li contributed equally to this work.




\section{Cellular Physiology Cell Physiol Biochem 2018;47:2484-2497 \begin{tabular}{l|l} 
DOI: 10.1159/000491622, & $\begin{array}{l}\text { O 2018 The Author(s). Published by S. Karger AG, Basel } \\
\text { www.karger.com/cpb }\end{array}$
\end{tabular} \\ Wei et al.: SKP2 Promotes HCC Through Nuclear Autophagic Regulation}

\section{Introduction}

Hepatocellular carcinoma (HCC) is the sixth most frequent tumor with an accelerating incidence in recent years. Over 750, 000 new cases are reported per year worldwide [13]. Approximately $10 \%$ to $15 \%$ have a five-year survival rate, marking this malignancy as the third most lethal cancer [4]. Efforts are being made to find novel target molecules which can be used to ameliorate HCC treatment. Autophagy, a conserved self-cannibalistic catabolic process, has gained attention recently as an essential contributor to cell survival, differentiation, development, and homeostasis [5]. Signaling from the crucial nutrientsensitive kinases mTORC1 and adenosine monophosphate-activated protein kinase (AMPK) forms the major hub in autophagy-related pathways. After energy deprivation, numerous signaling pathways inhibiting mTORC1 activity then induce autophagy and AMPK is a well-established negatively-regulated upstream signal of mTORC1. AMPK can also directly activate autophagy, leading to direct ULK1 activation [6, 7]. Autophagy has well-established tumor-suppressive properties. Although a large and increasing amount of researchers have been shed light on the role of autophagy in cancer, it is still unclear whether autophagy acts as a tumor suppressor or protects cancer cells from anti-cancer therapy-induced destruction or unfavorable conditions $[8,9]$. Additionally, our understanding mostly focuses on the molecular mechanisms that the nuclear event in autophagy regulation and is still quite primitive $[9,10]$.

SKP2 (S-phase kinase-associated protein 2), is a component of the SCF (SKP1-Cullins-F box protein complex) ligase, which is one of the RING-finger type ubiquitin ligases belonging to cullin RING ubiquitin ligases (CRLs). The functions of (SKP2 or CRLs) as both direct regulators of cyclin-dependent kinases (CDKs) and gene transcription regulators have been well-characterized $[11,12]$. SKP2 always acts as a classic oncogene by posttranscriptional regulation of oncosuppressors and functions via cell cycle related mechanisms in a variety of human cancers [13-16]. The previous HCC studies about SKP2 have paid more attention on CKS1/SKP2 as the cell cycle regulator or SKP2 negatively correlation to tumor suppressors that might be a diagnostic or therapeutic target $[14,17,18]$. Recent studies in some cell lines have found that in the process of energy deprivation induced autophagy AMPK phosphorylates FOXO3a in the nucleus, which in turn transcriptionally inhibits SKP2. CARM1 and TFEB transcriptionally co-activates on autophagy and lysosomal related genes. But SKP2-containing SCF E3 ubiquitin ligase is responsible for ubiquitination and proteasomal degradation of CARM1 in the nucleus when nutrients are sufficient [19]. Therefore, it is of great interest to address whether down-regulation of SKP2 inactivates degradation of CARM1, namely, the stabilization of CARM1 in the nucleus under energy deprived conditions, and its effects on autophagy in the progression of HCC.

In this study, we are focusing on identifying the levels of SKP2 in HCC cell lines and tissues, deciphering its biological function of SKP2 in HCC progression and maintenance, and confirming whether the nuclear AMPK-SKP2-CARM1 signaling of autophagy induction takes place in HCC while exploring its superficial mechanism, thereby opening a new horizon into comprehending the complexity of SKP2 in HCC tumorigenesis and possibly offering potential targets for HCC therapy.

\section{Materials and Methods}

Tissue samples and cell lines

HCC and adjacent normal liver tissues were obtained from a total of 72-paired hepatectomy patients at The First Affiliated Hospital of Nanjing Medical University from Jan. 2nd, 2017 to Jul. 1st, 2017. The tissues were all immediately stored in liquid nitrogen and the patients' diagnoses were clarified and the pathological types were affirmed independently by at least two professional pathologists. The study involved enrolled patients with the approval of Ethics Committee of the Nanjing Medical University and all participants signed written documents of informed consent. The human HCC cell lines HepG2, Hep3B, 


\section{Cellular Physiology Cell Physiol Biochem 2018;47:2484-2497 \begin{tabular}{ll|l} 
DOI: 10.1159/000491622, & $\begin{array}{l}\text { O 2018 The Author(s). Published by S. Karger AG, Basel } \\
\text { www.karger.com/cpb }\end{array}$
\end{tabular} \\ Wei et al.: SKP2 Promotes HCC Through Nuclear Autophagic Regulation}

SMMC-7721, MHCC97H, and Huh7, and the normal human hepatic cell line (LO2) were purchased from the Chinese Academy of Sciences (Shanghai, China) and cultured with Dulbecco's modified Eagle's medium (DMEM; Gibco, Thermofisher Scientific Inc., USA) with the addition of $10 \%$ fetal bovine serum (FBS; Gibco, Thermofisher Scientific Inc., USA) and $1 \%$ antibiotics (100 U/ml penicillin G and $100 \mathrm{mg} / \mathrm{ml}$ streptomycin) at $37^{\circ} \mathrm{C}$ with $5 \% \mathrm{CO}_{2}$ in a cell incubator (Thermofisher Scientific Inc., USA). All cell lines were regularly tested for mycoplasma contamination. For glucose starvation, cells were washed with PBS three times, then incubated with glucose-free DMEM supplemented with 10\% FBS and 1\% antibiotics for specific time periods.

\section{Quantitative polymerase chain reaction ( $q P C R$ )}

TRIzol (Invitrogen, USA) was used to isolate the total RNA of tissues and cells, and cDNA was synthesized with PrimeScript ${ }^{\mathrm{TM}}$ RT Master Mix (Takara Biomedical Technology Co., Ltd, Beijing). A 7900 Real-Time PCR System (Applied Biosystems, USA) with AceQ qPCR SYBR Green Master Mix (High ROX Premixed) (Vazyme Biotech Co., Ltd, Nanjing) was used to perform qPCR. Relative mRNA expression was standardized to the housekeeping gene $\beta$-actin forward: $5^{\prime}$ - ATTGCCGACAGGATGCAGAA- $3^{\prime}$ reverse: $5^{\prime}$ - ACATCTGCTGGAAGGTGGACAG-3'. The following human primers were used in this study: SKP2 forward: 5'-ATGCCCCAATCTTGTCCATCT-3' reverse: 5'-CACCGACTGAGTGATAGGTGT-3'; AMPKA1

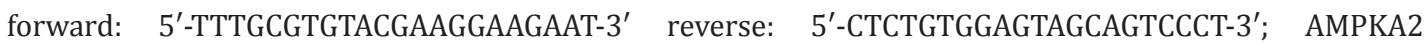
forward: $5^{\prime}$ - CTGTAAGCATGGACGGGTTGA-3' reverse: $5^{\prime}$ - AAATCGGCTATCTTGGCATTCA-3'; ATG12 forward: 5'- CTGTGTAATTGCGTCCCCCT-3' reverse: 5'- CACGCCTGAGACTTGCAGTA-3'; ATG14 forward: 5'AAAATCCCACGTGACTGGCT-3' reverse: 5'- CGATAAACCTCTCCCGGTCG-3'. The procedures were performed in triplicate.

\section{Lentivirus transfection}

SKP2 siRNA/shRNA/RNAi Lentivirus (Human) (piLenti-siRNA-GFP) (Applied Biological Materials (abm) Inc., Canada) and Scrambled siRNA GFP Lentivirus (Applied Biological Materials (abm) Inc., Canada), as a control, were transfected into cells according to the manufacturer's instructions and then verified by qPCR and Western blot. Two of the target sequences that proved the most effective for knockdown of gene expression were selected, Target-a: 846 AGAGACCATCACCCAGCTGAATCTTAGCG and Target-b: 1158 ACAGATTAATTGCTCCCATTTCACCACCA. SKP2 Lentivirus (Human) (CMV) (pLenti-GIII-CMV-GFP-2A-Puro) (Applied Biological Materials (abm) Inc., Canada) was used to overexpress SKP2 and verified by qPCR and Western blot. Viral liquid and $5 \mu \mathrm{g} / \mathrm{ml}$ polybrene (Genepharma, Shanghai), which increases the infection efficiency, were used to infect the target cells at $80 \%$ confluence according to the manufacturer's instructions. Stable cell lines were selected using $7 \mu \mathrm{g} / \mathrm{ml}$ puromycin (Sigma-Aldrich, USA) for 1 week.

\section{Antibodies and Western blot}

The following commercially available antibodies were used: Anti-SKP2 (\#2652), anti-CARM1 (\#3379), anti-LC3 (\#2775), anti-SQSTM1/p62 (\#5114), anti-AMPKa2 (\#2757), anti- $\beta$-Actin (\#4970) and Anti-rabbit IgG, HRP-linked Antibody (\#7074), all from Cell Signaling Technology, Inc., USA, while anti-Lamin A/C (10298-1-AP) was from Proteintech Group (Wuhan, China).

Cells were washed with ice-cold PBS before collection. For whole cell lysates, cells were lysed with RIPA buffer (Beyotime, Shanghai, China) supplemented with PMSF, protease inhibitors and resuspended, then sonicated by Sonifier (Branson Ultrasonics, USA). For cytosolic and nuclear fractions, a Nuclear and Cytoplasmic Protein Extraction Kit (KeyGEN BioTECH, Nanjing, China) was used according to the manufacturer's instructions. All lysates were quantified using a Bradford protein assay kit (Beyotime, Shanghai, China) and analyzed using $8 \%$ or $15 \%$ SDS-PAGE (Bio-Rad, USA). The proteins were transferred to PVDF membranes (Merck, Germany) and incubated with specific primary antibodies (1:1000), slightly shaking at $4^{\circ} \mathrm{C}$ overnight after blocking in skim milk for $2 \mathrm{~h}$ at room temperature. After washing with TBST (TBS- Tween-20), the membranes were incubated with HRP-conjugated anti-rabbit IgG antibodies (1:5000) at room temperature for $2 \mathrm{~h}$. Immunoreactive bands were visualized using an enhanced chemiluminescence detection system (BioRad, USA).

Cell Counting Kit-8 assays and colony formation assays

Cell viability was assessed using the Cell counting Kit- 8 assay (Dojindo Molecular Technologies, Inc., Shanghai, China). Cells were seeded in 96-well plates at a density of $5 \times 10^{3}$ cells in $100 \mu \mathrm{L}$ DMEM or glucose- 


\section{Cellular Physiology Cell Physiol Biochem 2018;47:2484-2497 \begin{tabular}{l|l} 
DOI: 10.1159/000491622, & $\begin{array}{l}\text { O 2018 The Author(s). Published by S. Karger AG, Basel } \\
\text { www.karger.com/cpb }\end{array}$
\end{tabular} \\ Wei et al.: SKP2 Promotes HCC Through Nuclear Autophagic Regulation}

free DMEM per well. For the colony formation assay, HepG2, Hep2, Hep3B, SMMC- 7721, and MHCC97H, at a density of 500 cells, and L02, at a density of $1 \times 10^{3}$ were cultured in $6 \mathrm{~mm}$ plates. The cells were allowed to adhere and colonize for two weeks to visualize colonies. The culture medium was then removed, the cells were fixed with methanol for $15 \mathrm{~min}$., and then stained with $0.1 \%$ crystal violet staining solution at $37^{\circ} \mathrm{C}$ for $30 \mathrm{~min}$.

\section{Cell migration and invasion assays}

The $6.5 \mathrm{~mm}$ chambers with $8 \mu \mathrm{m}$ pores (Millipore, Germany) in the 24-well plates were used to performed cell migration and invasion assays. For migration assays, the top chamber was seeded by suspending, separately, HepG2, Hep3B, SMMC-7721, MHCC97H, and Huh7 cells at a density of $1 \times 10^{5}$ and L02 cells at a density of $1 \times 10^{6}$ in serum-free DMEM or serum-free and glucose-free DMEM for glucose deprivation. Thereafter, $600 \mu \mathrm{L}$ of $10 \%$ complete DMEM or glucose-free DMEM for glucose deprivation was added to the lower chamber. This migration system was plated in $5 \% \mathrm{CO} 2$ at $37^{\circ} \mathrm{C}$ for $24 \mathrm{~h}$. For invasion assays, a chamber was seeded by suspending, separately, HepG2, Hep3B, SMMC-7721, MHCC97H, and Huh7 cells at a density of $1 \times 10^{5}$ and L02 cells at a density of $1 \times 10^{6}$ with Matrigel Matrix basement membranes (Corning, USA) coated and incubated for $24 \mathrm{~h}$ in $5 \% \mathrm{CO} 2$ at $37^{\circ} \mathrm{C}$. After incubation, the cells were fixed with $4 \%$ paraformaldehyde for $15 \mathrm{~min}$, stained with $0.1 \%$ crystal violet staining solution at $37^{\circ} \mathrm{C}$ for $30 \mathrm{~min}$, washed with PBS, then removed with cotton swabs. Cells were imaged using a light microscope at $20 \times$ magnification in four randomly taken fields. The assays were conducted in triplicate.

\section{Immunohistochemistry}

Tissues were fixed in 4\% paraformaldehyde, embedded in paraffin, dehydrated in isopropyl alcohol, and the alcohol was removed by xylene. Standard staining with hematoxylin and eosin was performed on $5 \mathrm{~mm}$ thick sections from each specimen block. These sections were deparaffinized and incubated in citrate buffer at $95^{\circ} \mathrm{C}$ for $40 \mathrm{~min}$ for antigen retrieval and then incubated overnight at $4^{\circ} \mathrm{C}$ with the primary antibodies. After three washes with PBS, tissue sections were incubated with secondary antibody for $1 \mathrm{~h}$ at room temperature and the slides incubated for $45 \mathrm{~min}$ after three washes. DAB solution (Vector Laboratories, USA) was added and the slides were counterstained with hematoxylin. Three fields of the tissue slices were selected at random to examine the percentage of positive tumors and staining intensities using Image J Software (National Institutes of Health, USA).

IHC staining scores was calculated as previous reported [20]. Staining intensity grades were divided as: 0 , none; 1 , weak; 2 , moderate; 3 , strong. The percentage of positive staining tumor cells were classified as: $0(<5 \%), 1(6 \%-25 \%), 2(26 \%-50 \%), 3(51 \%-75 \%)$, and $4(>75 \%)$. Finally, the scores were exhibited as the staining intensity multiplied by the percentage of specifically positive staining tumor cells [20].

\section{Immunofluorescence}

Dissociated cells were seeded onto coverslips at a density of $5 \times 10^{5}$, washed with PBS three times, and incubated with glucose-free DMEM for $0 \mathrm{~h}, 6 \mathrm{~h}, 12 \mathrm{~h}$, or $24 \mathrm{~h}$ after adhering. Cells transfected with lentivirus were directly fixed after adhering. All cells were fixed by $4 \%$ paraformaldehyde for $15 \mathrm{~min}$, permeabilized with $0.1 \%$ Triton $^{\mathrm{TM}} \mathrm{X}-100$ for $10 \mathrm{~min}$ at room temperature, blocked with 3\% BSA for $1 \mathrm{~h}$, and incubated with primary antibodies at $4^{\circ} \mathrm{C}$ overnight. The following day, the cells were washed with PBS, incubated with fluorochrome-conjugated secondary antibodies (Cell Signaling Technology, Inc. Anti-rabbit \#4412) for $1 \mathrm{~h}$ at room temperature in the dark, then washed with PBS and co-stained with DAPI (Cell Signaling Technology, Inc. CST \#8961). All pictures were taken with a fluorescence microscope (Zeiss, Scope.A1, Germany). HCC specimen slides were prepared in the same manner.

\section{Statistical analysis}

The data were reported as means \pm standard deviations and analyzed by IBM SPSS Statistics 23.0 Software (USA). Qualitative data were analyzed using the $X^{2}$ test. The differences between two groups were analyzed using the unpaired two-sided Student's t test. The differences among more than two groups were analyzed using one-way ANOVA with Bonferroni post-hoc tests. $P$ values $<0.05$ were considered statistically significant. 


\section{Cellular Physiology \begin{tabular}{ll|l} 
and Biochemistry Published online: July 10, 2018 & $\begin{array}{l}\text { (c) } 2018 \text { The Author(s). Published by S. Karger AG, Basel } \\
\text { www.karger.com/cpb }\end{array}$ \\
\hline
\end{tabular}

\section{Results}

SKP2 expression is significantly increased in HCC tissues and cell lines

To investigate the role of SKP2 in HCC tissues, the level of SKP2 was examined in 72 pairs of human HCC with corresponding adjacent paracancerous tissues by quantitative PCR (qPCR). As shown in Fig. $1 \mathrm{~A}$, the expression of SKP2 was significantly higher in HCC than in adjacentparacancerous tissues. Next, we detected the SKP2 expression using immunohistochemistry (IHC) staining analysis (Fig, 1B and 1C). Higher SKP2 levels were detected in the nucleus of tumor cells, while the adjacent paracancerous tissues. Meanwhile, the immunofluorescence (IF) results were consistent with the IHC analysis, which also showed stronger SKP2 expression in the nucleus of HCC tissues (Fig. 1D). We then analyzed the relationship between SKP2 levels and the clinicopathological features of HCC. The 72 cases HCC patients were divided into two groups: a high SKP2 group which showed more SKP2 expression in HCC tissues than adjacent paracancerous tissues, and a low SKP2 group which had less SKP2 expression in HCC tissues compared with adjacent paracancerous tissues. The high SKP2 group consisted of 55 cases $(76.4 \%)$ of the total 72 pairs investigated, while 17 cases (23.6\%) were in the low SKP2 group (Table 1). Higher SKP2 expression was statistically associated with a greater Edmondson-Steiner grade, larger tumor diameter, and greater intra-hepatic metastases. No significant relationship was found between SKP2 expression and other clinical features, including age, gender, tumor vascular invasion, alpha-fetoprotein level, HBV infection, or liver cirrhosis. These results indicated that the SKP2 gene was upregulated in HCC tissues, and was probably responsible for poor tumor differentiation and metastases in HCC patients.

To determine whether the expression of SKP2 was different in HCC cell lines HepG2, Hep3B, MHCC97H, Huh7, and SMMC-7721 compared with the human hepatic cell line L02, qPCR and Western blots were carried out as shown in Fig. 1E and Fig, 1F. Higher SKP2 expression was discovered in several HCC cell lines relative to the normal hepatic cell line L02 in mRNA and protein levels.

\section{Autophagy occurs after glucose deprivation}

To explore the role of SKP2 in autophagic processes and the effects of glucose deprivation-induced autophagy in HCC progression, we induced autophagy in HCC cell lines

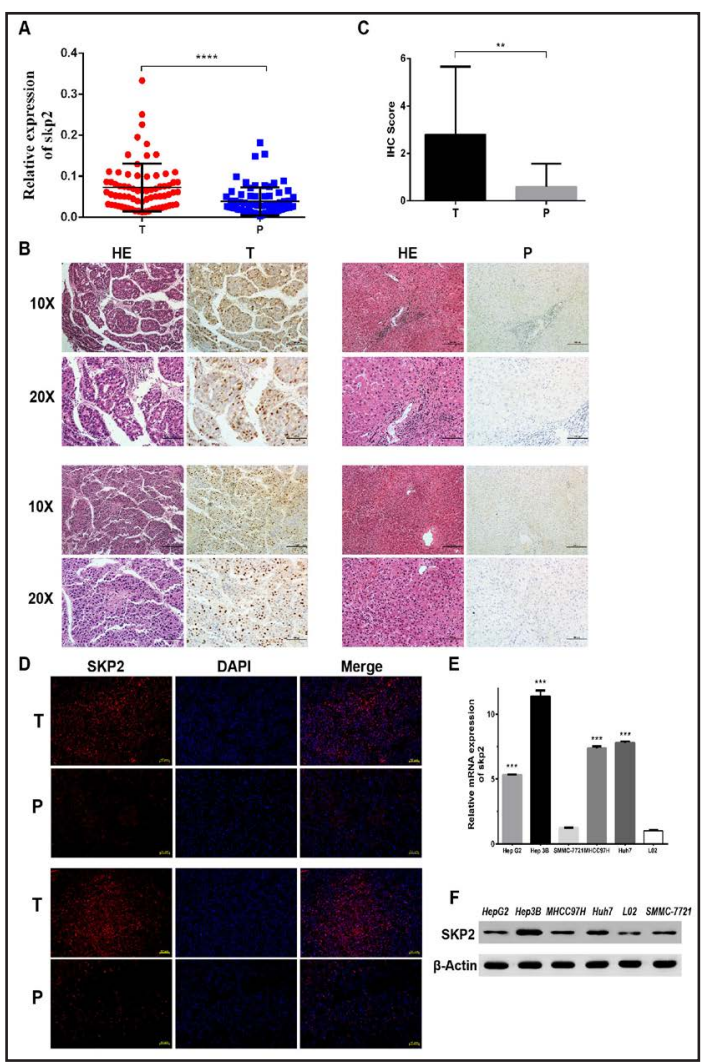

Fig. 1. The expression levels of SKP2 in HCC tissues and cell lines. (A) The SKP2 levels were detected in 72 pairs of human HCC (T) with corresponding adjacent paracancerous tissues (P) by qPCR. (B) Representative pictures of SKP2 IHC staining in HCC with adjacent paracancerous tissues. (C) Quantitative analysis of 72 HCC with its adjacent paracancerous samples SKP2 staining (IHC Scores). (D) Representative IF images of SKP2 in HCC and paracancerous tissues. (E) The SKP2 mRNA expressions in HCC cell lines HepG2, Hep3B, MHCC97H, Huh7, SMMC-7721, and the non-tumorigenic human cell line LO2 were examined by qPCR. (F) The SKP2 protein levels in HCC cell lines and the normal hepatic cell line L02. All the qPCR and Western blot data were collected in triplicate, and they are presented as mean \pm S.D. values. ${ }^{* *} \mathrm{P}<0.01,{ }^{* * *} \mathrm{P}<0.001,{ }^{* * * *} \mathrm{P}<0.0001$. 
by culturing them in glucosefree DMEM. LC3B, one of the widely used isoforms of LC3 located in the cytoplasm, and which is a common marker of autophagosomes [21], increased with glucose deprivation in HCC cell lines (Fig. 2A). The relative mRNA level of autophagy-related genes, ATG12 and ATG14, was up-regulated after glucose starvation (Fig. 2B). As is shown in Fig. 2C, autophagic flux was analyzed using the conversion from endogenous LC3-I to LC3-II and levels of P62 (also known as SQSTM1), which selectively incorporated into autophagosomes through direct binding to LC3, causing preferential and efficient degradation by autophagy. Thus, the total cellular expression levels of p62 inversely correlate with autophagic activity, making p62 levels a well-established parameter of autophagic flux [21]. In accordance with previous immunofluorescence results, glucose starvation accelerated the LC3-I to LC3-II conversion and P62 degradation. Thus, autophagy could be triggered by glucose deprivation and developed as glucose deficient time increased in HCC cell lines.

The expression of SKP2 and CARM1 is affected by glucose starvation induced autophagy

Since CARM1 is a crucial component of autophagy and its stability is regulated by SKP2 under nutrient-rich conditions in the nucleus [19], we then sought to monitor the level of SKP2 and CARM1 in the nucleus and cytoplasm, respectively, after glucose deprivation in HCC cell lines at different time intervals. A reduction of SKP2 and an augmentation of CARM1 protein levels were observed at multiple time segments under nutrition starved conditions (Fig. 3A and 3B). Besides, the SKP2 mRNA level was down-regulated after autophagy was induced (Fig. 3C). Notably, nutrient starvation resulted in increased levels of the CARM1 protein and decreased SKP2, which was more obvious in the nucleus than in the cytoplasm of HCC cells with induced autophagy (Fig. 3D).

Knockdown or overexpression of SKP2 influences the nuclear level of CARM1 under nutrient-rich conditions

To elucidate whether SKP2 regulated the CARM1 protein level in the nucleus of HCC cells under nutrient-rich conditions, we used the SKP2 siRNA lentivirus system with two targets (defined as siSKP2-a and siSKP2-b), which were identified to silence SKP2 expression and induce the SKP2 lentivirus to overexpress SKP2 in HCC cell lines. The GFP lentivirus was transfected simultaneously as a control group. SKP2 siRNA was transfected into HepG2, Hep3B, MHCC97H, and Huh7, which had relatively higher SKP2 levels, and L02 as a control. SKP2 was overexpressed in SMMC-7721 and L02 cells which had relatively lower endogenous SKP2 levels. The siSKP2 and SKP2-Overexpression HCC cell lines were stably established and 
Fig. 2. Autophagy is induced by glucose deprivation. (A) IF images of LC3 in glucose deprived (Glu.dep) HCC cells. HepG2, Hep3B, MHCC97H, Huh7, and L02 cells were glucose-starved for $0 \mathrm{~h}, 6 \mathrm{~h}, 12 \mathrm{~h}$, and 24 h. (B) The mRNA expression of autophagy-related genes, ATG12 and ATG14, was detected in HepG2, Hep3B, MHCC97H, Huh7 and L02 cells, which were glucosestarved for $0 \mathrm{~h}, 6 \mathrm{~h}, 12 \mathrm{~h}$, and $24 \mathrm{~h}$, separately. (C) LC3-I to LC3-II conversion and P62, the symbol of autophagic flux activity, were monitored by Western blot in HCC cell lines at $0 \mathrm{~h}, 6 \mathrm{~h}, 12 \mathrm{~h}$, and $24 \mathrm{~h}$ of their glucose-deficient time. All the qPCR and Western blot data were collected in triplicate, and the data are presented as mean \pm S.D. values. ${ }^{* *} \mathrm{P}<0.01$.

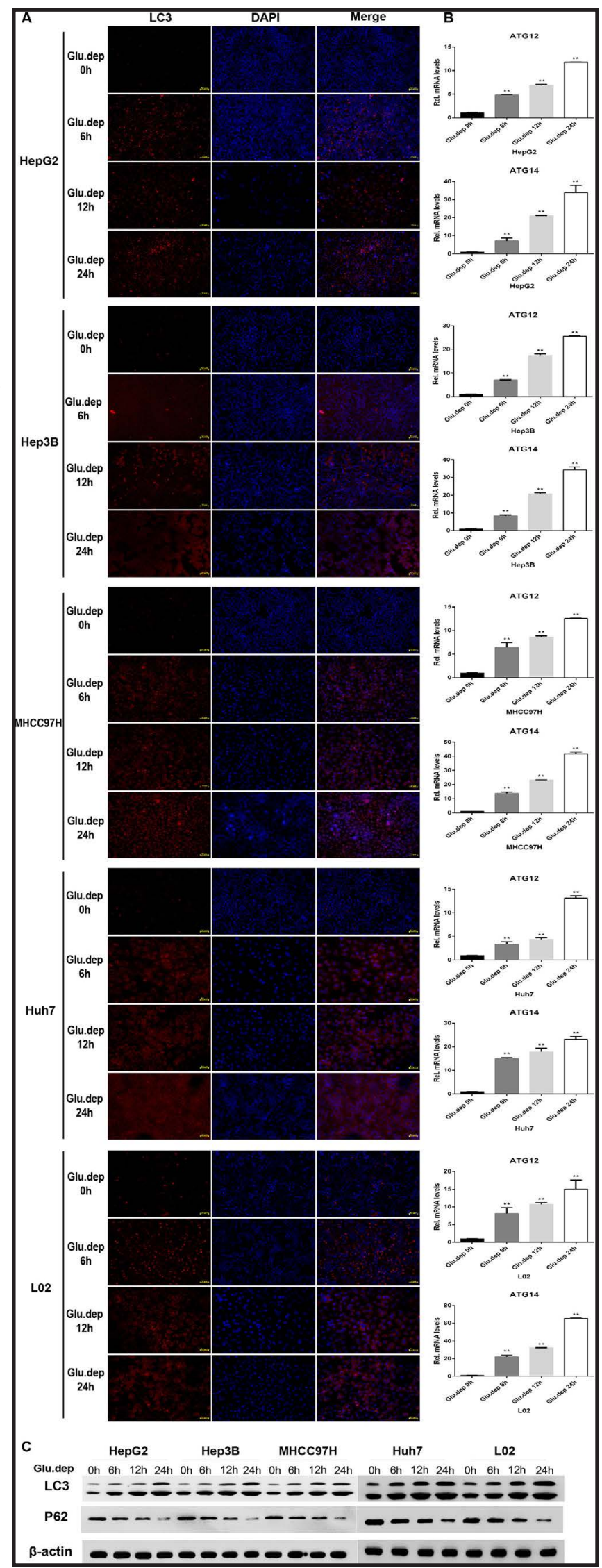




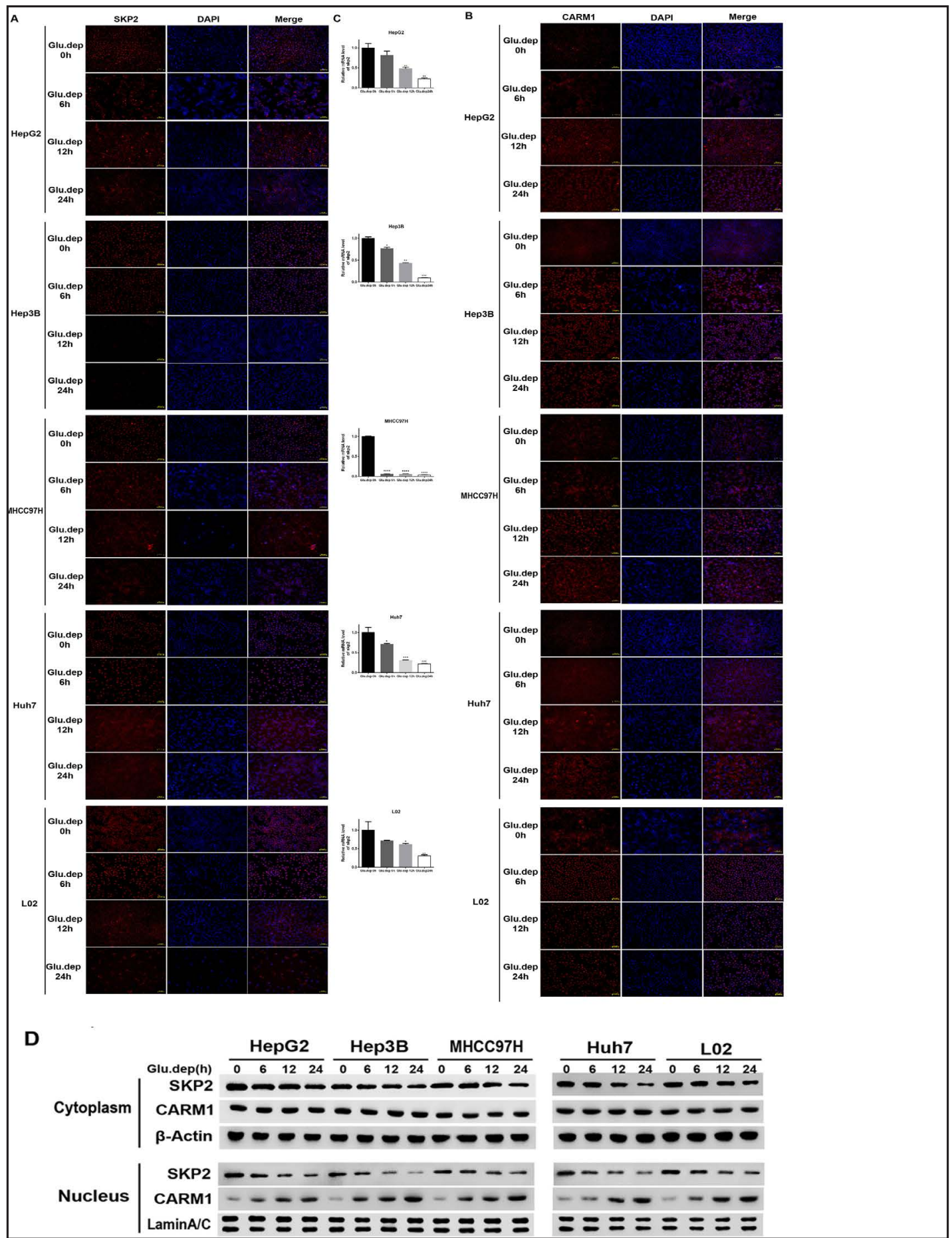

Fig. 3. Glucose starvation induced autophagy impacts on SKP2 and CARM1 levels. (A) The SKP2 levels of glucose-deprived (Glu.dep) HCC cells; HepG2, Hep3B, MHCC97H, Huh7 and L02 at 0 h, 6 h, 12 h, and 24 h were monitored by IF. (B)The CARM1 levels after glucose-deprived separately for $0 \mathrm{~h}, 6 \mathrm{~h}, 12 \mathrm{~h}$, and $24 \mathrm{~h}$ in HepG2, Hep3B, MHCC97H, Huh7 and L02 cells were detected by IF. (C) qPCR was used to analyze the SKP2 mRNA expression in HepG2, Hep3B, MHCC97H, Huh7 and L02 cells which were glucose-deprived (Glu.dep) for $0 \mathrm{~h}, 6 \mathrm{~h}, 12 \mathrm{~h}$, and $24 \mathrm{~h}$. (D) The cytoplasmic and nuclear SKP2 and CARM1 protein levels of HCC cell lines HepG2, Hep3B, MHCC97H, Huh7 and L02 which were glucose-deprived for 0 h, 6 h, 12 h, and 24 h. All the qPCR and Western blot data were collected in triplicate, and the representative data are displayed as mean \pm S.D. values. ${ }^{*} \mathrm{P}<0.05,{ }^{* *} \mathrm{P}<0.01,{ }^{* * *} \mathrm{P}<0.001$.

\section{KARGER}


verified at the mRNA and protein level by qPCR and Western blots (Fig. 4A and 4B, Fig. 4E and 4F). Knockdown of SKP2 led to significantly increased CARM1 protein levels in the nucleus of Hep3B, MHCC97H, and L02 (Fig. 4C). Conversely, SKP2 was overexpressed in SMMC-7721 and L02, represented by lower CARM1 nuclear protein levels (Fig. 4F). Furthermore, the immunofluorescence assays in the Hep3B, MHCC97H, and L02 SKP2 knockdown systems validated that the siSKP2 groups had higher CARM1 levels in the nucleus than the blank or GFP groups. This was in accordance with Western blot assays (Fig. 4D).

Fig. 4. Down-regulation or up-regulation of SKP2 alters CARM1 nuclear levels. (A) mRNA levels of SKP2 detected by qPCR in the SKP2 siRNA lentivirus-transfected HCC cell lines HepG2, Hep3B, MHCC97H, Huh7, L02 and the SKP2 knockdown stable cell lines siSKP2-a, siSKP2-b with blank, and control GFP groups. (B) Protein levels of SKP2 as detected by Western blot. (C) CARM1 levels in the nucleus after SKP2 knockdown (siSKP2). (D) IF was used to analyze the CARM1 levels in Hep3B, MHCC97H, and L02 siSKP2 groups compared to blank and GFP groups. (E) SMMC-7721 and L02 cells were transfected with SKP2 lentivirus to establish a stable SKP2 overexpression cell line and confirmed with blank and GFP control groups by qPCR, and (F) Western blot, additionally, the CARM1 nuclear levels were detected in the SKP2 overexpressed cells. All the qPCR and Western blot data were collected in triplicate and the data are presented as mean \pm S.D. values. ${ }^{* *} \mathrm{P}<0.01$.

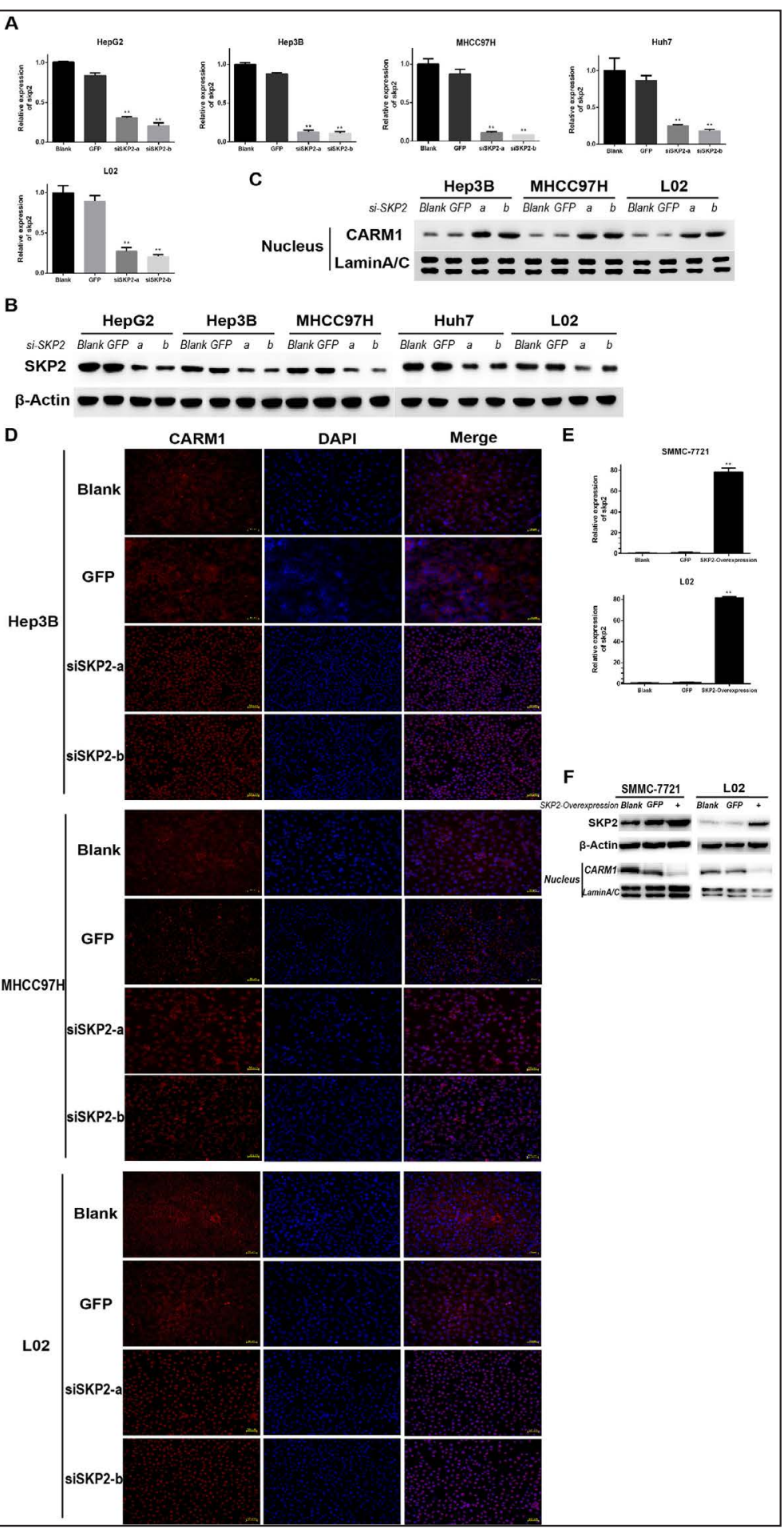


Fig. 5. The effects of SKP2 knockdown or overexpression and glucose deprivation (Glu.dep) on HCC cell proliferation, migration and invasion, and colony formation. (A) Cell viability of HepG2, Hep3B, MHCC97H, Huh7, and L02 blank cells, transfected with GFP, siSKP2-a, siSKP2b lentivirus, and glucose deprived cells were detected by CCK8 assays. (B) HepG2, Hep3B, MHCC97H, Huh7, and L02 cells were divided into blank, GFP, siSKP2-a, siSKP2-b, and glucosedeprived groups. The migration and invasion were evaluated by Transwell migration and Matrigel invasion assays in every group as previous. The migration and invasion assays were quantified as migrated or invaded cell numbers. (C) Cell viability of SMMC7721 and L02 blank cells, control GFP, SKP2 overexpression, and glucose deprived cells were examined by CCK8 assays. (D) The migration and invasion of SMMC-7721 blank, GFP, SKP2-Overexpression, glucose-deprived cells and L02, blank, GFP, and SKP2-Overexpression cells were detected by Transwell migration and invasion assays. The capabilities of migration and invasion were quantified as migrated or invaded cell numbers. (E) Representative colony formation images of Hep3B, MHCC97H, HepG2, blank, or SKP2 knockdown cells and SMMC-7721, L02, blank, or SKP2 overexpressed cells. All experiments were conducted in triplicate, and the data are presented as mean \pm S.D. values. ${ }^{*} \mathrm{P}<0.05,{ }^{* *} \mathrm{P}<0.01$.
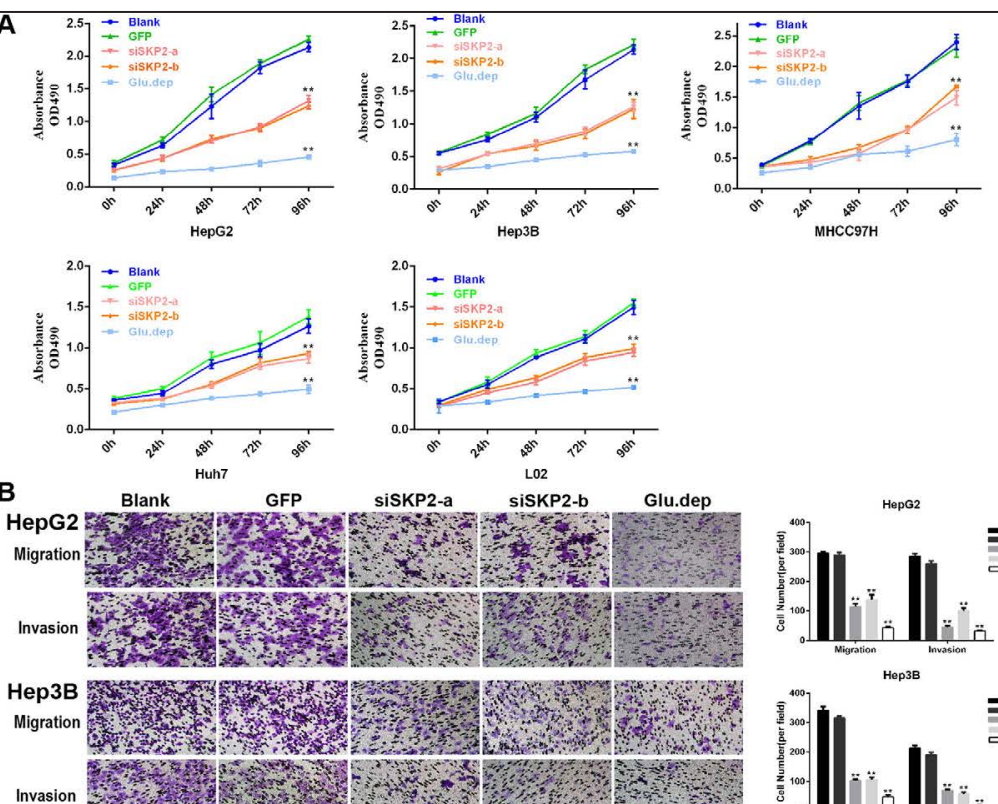

Invas
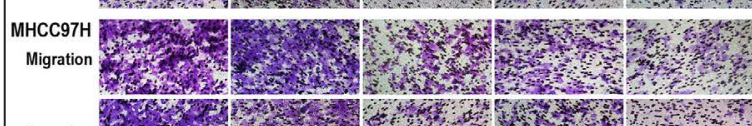

Invasion

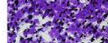

Huh7
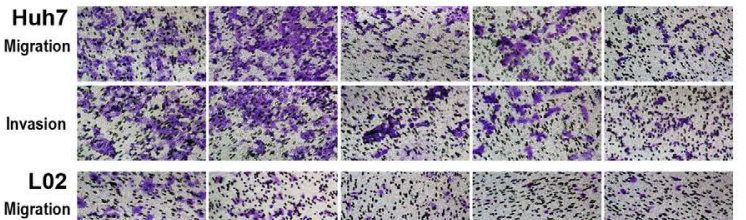

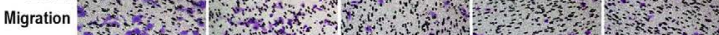
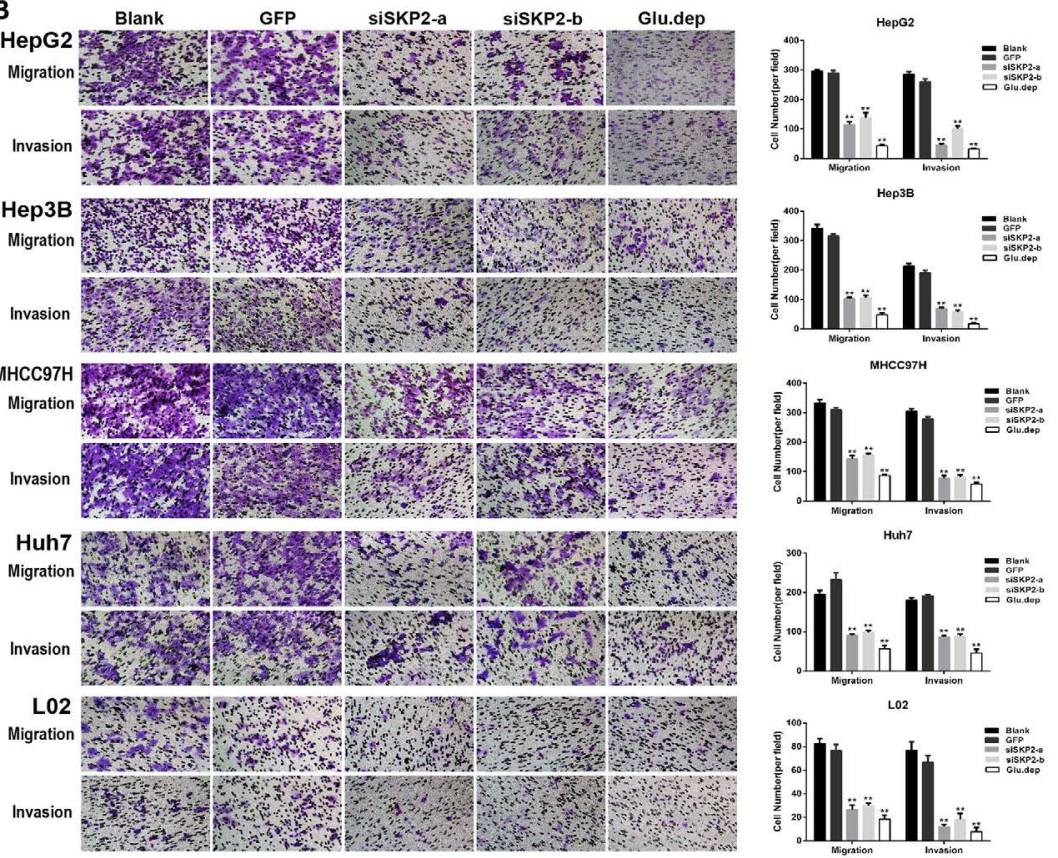

c
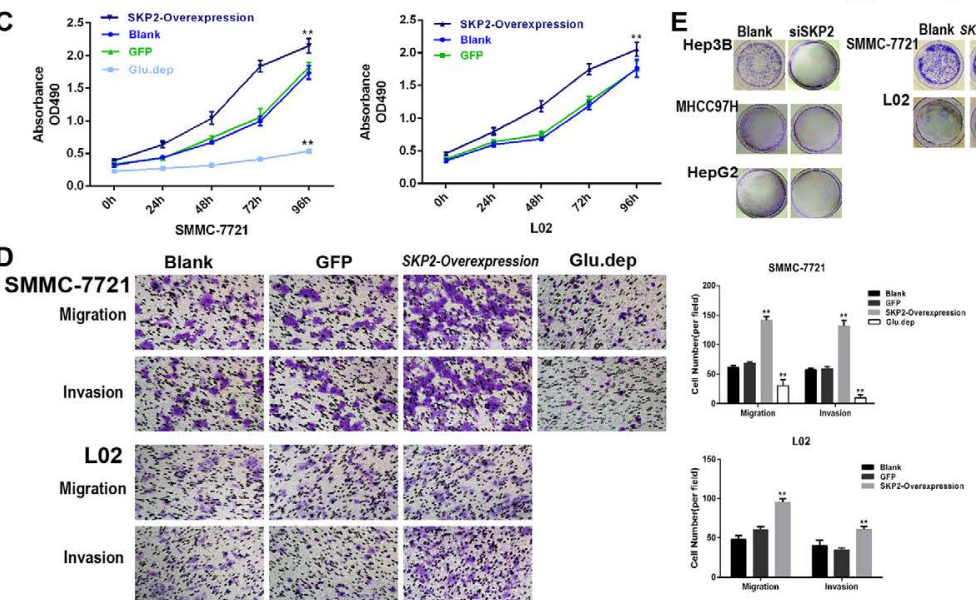
SKP2 facilitates HCC cell proliferation, migration, invasion and colony formation

To validate the biological function of SKP 2 in HCC, Cell Counting Kit-8, transwell migration and invasion assays and colony formation assays were conducted separately in blank, GFP, siSKP2, SKP2-Overexpression, and glucose deprived HCC cells. The cell viability was significantly inhibited when SKP2 was knocked down or under glucose deficient conditions (Fig. 5A). In addition, transwell migration and invasion assays showed that knockdown of SKP2 or deprivation of glucose significantly suppressed the migration and invasion of HCC cells (Fig. 5B). Furthermore, the colony formation images showed that colony growth was significantly decreased in the siSKP2 group cell lines (Fig. 5E). These observations suggest that down-regulation of SKP2 and glucose deprived condition suppress in vitro proliferation, migration and invasion of HCC. In contrast, in SMMC-7721 and L02 cells, SKP2-overexpression groups showed significantly increased cell proliferation, migration and invasion, and colony formation compared to the blank and GFP transfected cells (Fig. 5C-5E). These results demonstrated that SKP2 promotes several malignancy properties in human HCC cells.

\section{AMPKa2 accumulates in the nucleus when autophagy induced}

It has been reported that AMPK is activated during glucose starvation and leads to starvation-induced autophagy [22-24] Nutrient starvation causes AMP-activated protein kinase (AMPK)-dependent phosphorylation of FOXO3a in the nucleus, which in turn transcriptionally represses SKP2 [19]. Therefore, we aimed to evaluate whether AMPK inhibits SKP2 in the nucleus of HCC cells. Compared to AMPK $\alpha 1$, AMPK $\alpha 2$ mRNA levels were gradually, but significantly up-regulated as the process of glucose starved advanced

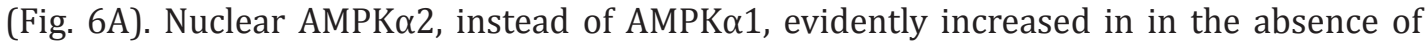
glucose (Fig. 6B). These data demonstrate that AMPK $\alpha 2$ is expressed in the nucleus of HCC cells under nutrient starved conditions, suggesting that it might perform a distinct role in the nucleus. Moreover, the HCC cells nuclear AMPK 22 accumulation has a possible role as the upstream signal resulting in the repression of SKP2 and stabilization of CARM1 after nutrient-deprivation induced autophagy.

Fig. 6. AMPK $\alpha 2$ is up-regulated in the nucleus when autophagy induced. (A) Relative mRNA levels of AMPK $\alpha 1$ and AMPK $\alpha 2$ in HepG2, Hep3B, MHCC97H, Huh7, and L02 in response to glucose-deprivation (Glu.dep) respectively at $0 \mathrm{~h}, 6 \mathrm{~h}$, $12 \mathrm{~h}$, and $24 \mathrm{~h}$ were analyzed by qPCR. (B) The protein levels of $A M P K \alpha 1$ and AMPK $\alpha 2$ in the nucleus were examined by western blot in the presence or absence of glucose. These experiments were conducted at least three times, and results are presented as mean \pm S.D. values. ${ }^{* *} \mathrm{P}<0.01$.

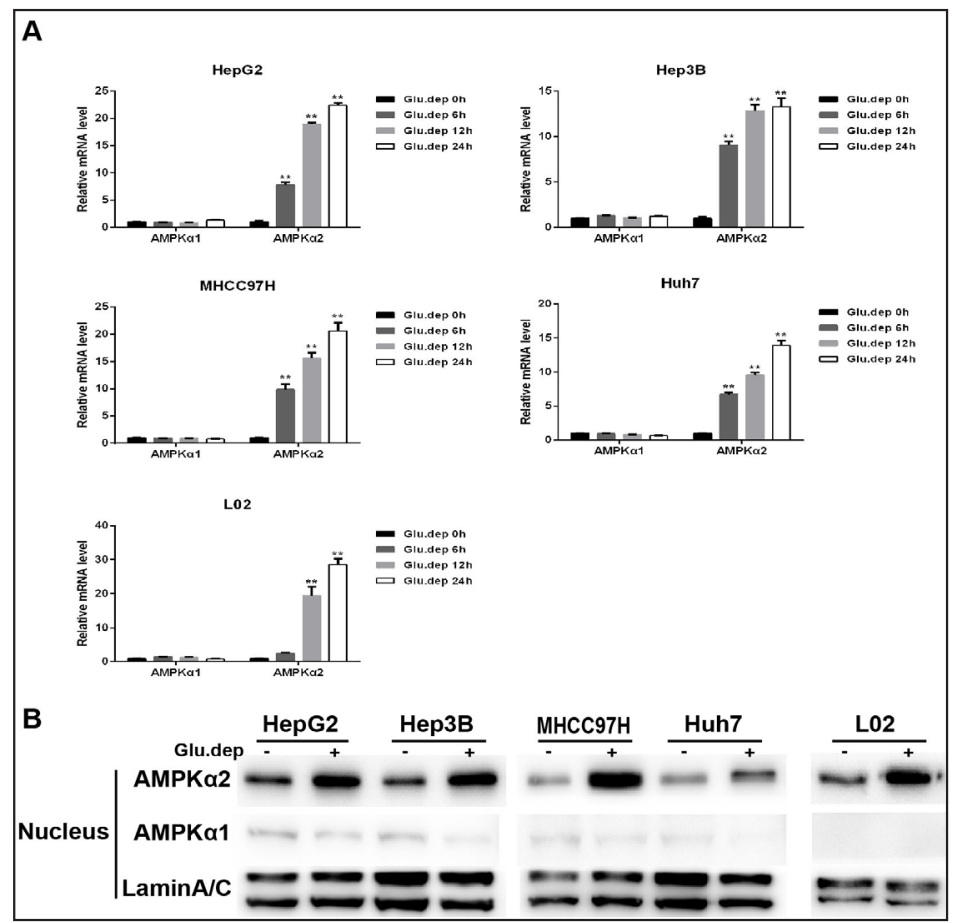




\section{Cellular Physiology Cell Physiol Biochem 2018;47:2484-2497 \\ \begin{tabular}{c|c} 
DOI: 10.1159/000491622 & $\begin{array}{l}\text { O } 2018 \text { The Author(s). Published by S. Karger AG, Basel } \\
\text { www.karger.com/cpb }\end{array}$
\end{tabular} \\ Wei et al.: SKP2 Promotes HCC Through Nuclear Autophagic Regulation}

\section{Discussion}

Various studies have shown that SKP2 exerts its tumorigenic characteristics through inhibiting tumor suppressor genes and their mediated pathways, repression of cyclindependent kinase inhibitors as tumor suppressor proteins [25-28]. As for HCC, there are still a number of questions about the oncogenic mechanism of SKP2. In this study, we demonstrated that SKP2 is significantly up-regulated in HCC tissue relative to normal liver tissues and HCC cell lines, and it promotes the proliferation, migration, invasion, and colony formation of HCC in vitro. SKP2 overexpression has been associated with poor prognosis in many human cancers [27-29]. In HCC patients, high levels of SKP2 have been shown to correlate with unfavorable outcomes [28]. We also found that the associations between SKP2 and HCC grades, tumor diameters, intra-hepatic metastases are statistically significant.

To our knowledge, autophagy has long been recognized as an important catabolism pathway, particularly during unfavorable conditions [7]. Although the effect of autophagy in cancer is still poorly understood, an increasing number of studies on autophagy and cancer indicate that autophagy appears paradoxical and may have different roles in tumor progression at different stages [30]. However, there have been few studies about the nuclear signaling or pathway of autophagy that acts on HCC advancement. In our study, we induced autophagy by glucose deprivation in HCC cell lines and L02, the human normal liver cell line, and it was validated by LC3- I to LC3- II conversion and p62 degradation. Furthermore, we discovered that autophagy induction inhibits proliferation, migration, invasion, and colony formation of HCC cell lines.

SKP2 was identified as a CARM1-specific binding protein along with cullin 1 (CUL1). Nuclear localization of SKP2 results in selective CARM1 ubiquitination in the nucleus under nutrient-rich conditions. Glucose starvation can markedly reduce the ubiquitination of CARM1 in the nucleus [19]. In line with this notion, we observed that down-regulation of SKP2 and augmented of CARM1 protein levels occur only in the nucleus after glucose deprivation in HCC cell lines. Furthermore, knockdown of SKP2 stabilized CARM1 is due to attenuated CARM1 ubiquitination in the nucleus. Conversely, overexpression of SKP2 accelerated the deficiency of CARM1 protein levels in the nucleus. Hence, we speculate that as a result of SKP2 down-regulation, the link between CUL1 and CARM1 is attenuated after glucose deprived. AMPK controls cell growth and energy metabolism by phosphorylating several substrates, including transcriptional factors in the FOXO family, and has been shown to be preferentially expressed in the nucleus [31]. In AMPK $\alpha 2$ knockout mice, the stimulatory function of the AMPK activator 5-aminoimidazole-4- carboxamide riboside (AICAR) on glucose uptake was lost. By contrast, in AMPK- $\alpha 1$ knockout mice, it was normal [32]. In support of their notions, we found that $A M P K \alpha 2$ increased in the nucleus after glucose starvation. In addition, it has been discovered in aged hearts that AMPK down-regulation gives rise to nuclear CARM1 deficiency mediated partly by SKP2, leading to autophagy disorders. In the nucleus, AMPK regulates CARM1 stabilization that controls autophagy of hearts [33]. These findings correlate with our hypothesis and results.

In summary, our study has identified that SKP2 promotes HCC progression and maintenance. We also confirm that in the nucleus, glucose deprivation induced autophagy is characterized by the decrease of SKP2, stabilization of CARM1, activation of AMPK $\alpha 2$, and when energy is sufficient, the nuclear CARM1 is inhibited by SKP2. Identification of nuclear AMPK-SKP2-CARM1 signaling of autophagy induction not only opens a new horizon into understanding the complex mechanism of SKP2 in HCC tumorigenesis, but also offers potentially therapeutic strategies for HCC.

\section{Acknowledgements}

This work was supported by grants from the National Natural Science Foundation of China (Grant Number: 81273262). 


\section{Cellular Physiology Cell Physiol Biochem 2018;47:2484-2497 and Biochemistry Published online: July 10, $2018 \quad \begin{aligned} & \text { DOI: 10.1159/000491622 } 2018 \text { The Author(s). Published by S. Karger AG, Basel } \\ & \text { www.karger.com/cpb }\end{aligned}$ \\ Wei et al.: SKP2 Promotes HCC Through Nuclear Autophagic Regulation}

\section{Disclosure Statement}

The authors declared no conflicts of interests.

\section{References}

$>1$ Armengol C, Sarrias MR, Sala M: Hepatocellular carcinoma: Present and future. Med Clin (Barc) 2018;150:390-397.

-2 Turato C, Balasso A, Carloni V, , Tiribelli C, Mastrotto F, Mazzocca A, Pontisso P: New molecular targets for functionalized nanosized drug delivery systems in personalized therapy for hepatocellular carcinoma. J Control Release 2017;268:184-197. Forner A, Llovet JM, Bruix J: Hepatocellular carcinoma. Lancet 2012;379:1245-1255.

Nault J-C, Sutter O, Nahon P, Ganne-Carrié N, Séror O: Percutaneous treatment of hepatocellular carcinoma: State of the art and innovations. J Hepatol 2017;17:32351-32356. Rabinowitz JD, White E: Autophagy and Metabolism. Science 2010;330:1344-1348. Russell RC, Yuan H-X, Guan K-L: Autophagy regulation by nutrient signaling. Cell Res 2013;24:42-57. Rubinsztein DC, Mariño G, Kroemer G: Autophagy Aging Cell 2011;146:682-695.

Kondo Y, Kanzawa T, Sawaya R, Kondo S: The role of autophagy in cancer development and response to therapy. Nature Rev Cancer 2005;5:726-734.

- Levine B, Kroemer G: Autophagy in the Pathogenesis of Disease. Cell 2008;132:27-42.

10 Baek K-H, Park J, Shin I: Autophagy-regulating small molecules and their therapeutic applications. Chem Soc Rev 2012;41:3245-3263.

11 Jackson PK, Eldrige AE: The SCF Ubiquitin Ligase: An extended look. Mol Cell 2002;9:923-925.

12 Chiariello M: Closing the Cycle: Skp2 Modulates Cyclic Nucleotides Antiproliferative Effects. Circ Res 2006;98:1113-1114.

13 Xu J, Zhou W, Yang F, Chen G, Li H, Zhao Y, Liu P, Li H, Tan M, Xiong X, Sun Y: The $\beta$-TrCP-FBXW2-SKP2 axis regulates lung cancer cell growth with FBXW2 acting as a tumour suppressor. Nature Commun 2017;8:14002.

14 Frescas D, Pagano M: Deregulated proteolysis by the F-box proteins SKP2 and $\beta$-TrCP: tipping the scales of cancer. Nat Rev Cancer 2008;8:438-449.

15 Gstaiger M, Jordan R, Lim M, Catzavelos C, Mestan J, Slingerland J, Krek W: Skp2 is oncogenic and overexpressed in human cancers. Proc Natl Acad Sci USA 2001;98:5043-5048.

16 Wang L, Ye X, Cai X, Su J, Ma R, Yin X, Zhou X, Li H, Wang Z: Curcumin suppresses cell growth and invasion and induces apoptosis by down-regulation of Skp2 pathway in glioma cells. Oncotarget 2015;6:1802718037.

17 Calvisi DF, Ladu S, Pinna F, Frau M, Tomasi ML, Sini M, Simile MM, Bonelli P, Muroni MR, Seddaiu MA, Lim DS, Feo F, Pascale RM: SKP2 and CKS1 Promote Degradation of Cell Cycle Regulators and Are Associated With Hepatocellular Carcinoma Prognosis. Gastroenterology 2009;137:1816-1826.

18 Zhang Y, Liu Y, Duan J, Yan H, Zhang J, Zhang H, Fan Q, Luo F, Yan G, Qiao K, Liu J: Hippocalcin-like 1 suppresses hepatocellular carcinoma progression by promoting p21Waf/Cip1stabilization by activating the ERK1/2-MAPK pathway. Hepatology 2016;63:880-897.

$>19$ Shin HJ, Kim H, Oh S, Lee JG, Kee M, Ko HJ, Kweon MN Won KJ, Baek SH: AMPK-SKP2-CARM1 signalling cascade in transcriptional regulation of autophagy. Nature 2016;534:553-557.

20 Zhang L, Jia G, Shi B, Ge G, Duan H, Yang Y: PRSS8 is Downregulated and Suppresses Tumour Growth and Metastases in Hepatocellular Carcinoma. Cell Physiol Biochem 2016;40:757-769.

-21 Mizushima N, Yoshimori T, Levine B: Methods in Mammalian Autophagy Research. Cell 2010;140:313-326.

22 Hardie DG: AMPK and autophagy get connected. EMBO J 2011;30:634-635.

23 Mihaylova MM, Shaw RJ: The AMPK signalling pathway coordinates cell growth, autophagy and metabolism. Nat Cell Biol 2011;13:1016-1023.

24 Inoki K, Kim J, Guan K-L. AMPK and mTOR in Cellular Energy Homeostasis and Drug Targets. Annu Rev Pharmacol Toxicol 2012;52:381-400.

-25 Ecker K, Hengst L:Skp2: caught in the Akt. Nature Cell Biol 2009;11:377-379. 


\section{Cellular Physiology Cell Physiol Biochem 2018;47:2484-2497 \begin{tabular}{l|l|l} 
DOI: 10.1159/000491622 & O 2018 The Author(s). Published by S. Karger AG, Basel \\
www.karger.com/cpb
\end{tabular} \\ Wei et al.: SKP2 Promotes HCC Through Nuclear Autophagic Regulation}

-26 Zhao H, He X, Pu Z, Wang Q: Expression of Skp2 and PTEN and its correlation in glottic carcinoma. Journal of Clinical Otorhinolaryngology, Head, and Neck Surgery 2008;22:688-691.

-27 Hulit J, Lee RJ, Li Z, Wang C, Katiyar S, Yang J, Quong AA, Wu K, Albanese C, Russell R, Di Vizio D, Koff A, Thummala S, Zhang H, Harrell J, Sun H, Muller WJ, Inghirami G, Lisanti MP, Pestell RG: p27Kip1 Repression of ErbB2-Induced Mammary Tumor Growth in Transgenic Mice Involves Skp2 and Wnt/ $\beta$-Catenin Signaling. Cancer Res 2006;66:8529-8541.

-28 Huang Y, Tai AW, Tong S, Lok AS.: HBV core promoter mutations promote cellular proliferation through E2F1-mediated upregulation of S-phase kinase-associated protein 2 transcription. J Hepatol 2013;58:10681073.

-29 Hershko DD: Oncogenic properties and prognostic implications of the ubiquitin ligase Skp2 in cancer. Cancer 2008;112:1415-1424.

-30 Levy JMM, Towers CG, Thorburn A: Targeting autophagy in cancer. Nature Rev Cancer 2017;17(9):528-42.

-31 Salt I, Celler JW, Hawley SA, Prescott A, Woods A, Carling D, Hardie DG: AMP-activated protein kinase greater AMP dependence, and preferential nuclear localization, of complexes containing the $\alpha 2$ isoform. Biochem J 1998;15:177-187.

32 Hardie DG, Ross FA, Hawley SA: AMPK: a nutrient and energy sensor that maintains energy homeostasis. Nature Rev Mol Cell Biol 2012;13:251-262.

33 Li C, Yu L, Xue H, , Yang Z, Yin Y, Zhang B, Chen M, Ma H: Nuclear AMPK regulated CARM1 stabilization impacts autophagy in aged heart. Biochem Biophys Res Commun 2017;486:398-405. 